\title{
University
}

Giles, J. T. et al. (2020) Cardiovascular safety of tocilizumab versus etanercept in rheumatoid arthritis: a randomized controlled trial. Arthritis and Rheumatology, 72(1), pp. 31-40.

This is the peer reviewed version of the following article: Giles, J. T. et al. (2020) Cardiovascular safety of tocilizumab versus etanercept in rheumatoid arthritis: a randomized controlled trial. Arthritis and Rheumatology, 72(1), pp. 31-40, which has been published in final form at http://dx.doi.org/10.1002/art.41095

This article may be used for non-commercial purposes in accordance with Wiley Terms and Conditions for Self-Archiving.

http://eprints.gla.ac.uk/195368/

Deposited on: 27 August 2019

Enlighten - Research publications by members of the University of Glasgow http://eprints.gla.ac.uk 
DR. JON TYLER GILES (Orcid ID : 0000-0002-8792-0402)

Article type : Full Length

\section{Cardiovascular Safety of Tocilizumab Versus Etanercept in Rheumatoid Arthritis: A}

\section{Randomized Controlled Trial}

Jon T. Giles, MD MPH, ${ }^{1}$ Naveed Sattar, FMedSci, ${ }^{2}$ Sherine Gabriel, MD, ${ }^{3}$ Paul M. Ridker, MD, ${ }^{4}$ Steffen Gay, MD, ${ }^{5}$ Charles Warne, MBiostat, ${ }^{6}$ David Musselman, MD, ${ }^{7}$ Laura Brockwell, MBiochem, ${ }^{6}$ Emma Shittu, PhD, ${ }^{6}$ Micki Klearman, $\mathrm{MD},{ }^{7}$ and Thomas R. Fleming, $\mathrm{PhD}^{8}$

${ }^{1}$ Division of Rheumatology, Columbia University College of Physicians \& Surgeons, New York, NY, United States; ${ }^{2}$ Institute of Cardiovascular and Medical Sciences, University of Glasgow, Glasgow, United Kingdom; ${ }^{3}$ Rutgers Robert Wood Johnson Medical School, New Brunswick, NJ, United States; ${ }^{4}$ Center for Cardiovascular Disease Prevention, Brigham and Women's Hospital, Harvard Medical School, Boston, MA, United States; ${ }^{5}$ Department of Rheumatology, University Hospital Zurich, Zurich, Switzerland; ${ }^{6}$ Roche Products Ltd., Welwyn Garden City, United Kingdom; ${ }^{7}$ Genentech, Inc., South San Francisco, CA, United States; ${ }^{8}$ Department of Biostatistics, University of Washington, Seattle, WA, United States

Running head: Cardiovascular Safety of Tocilizumab Versus Etanercept in Rheumatoid Arthritis

This article has been accepted for publication and undergone full peer review but has not been through the copyediting, typesetting, pagination and proofreading process, which may lead to differences between this version and the Version of Record. Please cite this article as doi: $10.1002 /$ art.41095

This article is protected by copyright. All rights reserved. 


\section{Corresponding author:}

Jon T. Giles, MD, MPH

Division of Rheumatology

Columbia University College of Physicians and Surgeons

630 West 168th Street

New York, NY 10032

Email: jtg2122@columbia.edu

Funding/Support: Supported by F. Hoffmann-La Roche Ltd. Support for third-party editorial assistance was funded by F. Hoffmann-La Roche Ltd.

Conflict of Interest Disclosures: Dr. Sattar reports receiving consulting fees and grant support from Boehringer Ingelheim, consulting fees from NovoNordisk, Janssen, and Eli Lilly, and grant support from AstraZeneca. Dr. Ridker reports receiving nonfinancial research support from AstraZeneca, Novartis, Roche, and Sanofi-Aventis. Charles Warne is an employee of Roche Products Ltd. Dr. Musselman is an employee of Roche Products Ltd. Laura Brockwell is an employee of Roche Products Ltd. Dr. Shittu is an employee of Roche Products Ltd. Dr. Klearman was an employee of Genentech at the time the study was conducted. Drs. Giles, Gabriel, Gay, and Fleming have nothing to disclose.

This article is protected by copyright. All rights reserved. 


\section{ABSTRACT}

Objective. To compare the risk for major adverse cardiovascular events (MACE) in RA patients treated with tocilizumab versus the tumor necrosis factor inhibitor etanercept. Methods. This randomized, open-label, parallel-group trial enrolled patients with active seropositive RA ( $N=3080)$, inadequate responses to conventional synthetic diseasemodifying antirheumatic drugs, and at least one cardiovascular risk factor. Patients were randomly assigned $1: 1$ to open-label tocilizumab $8 \mathrm{mg} / \mathrm{kg} / \mathrm{month}$ or etanercept $50 \mathrm{mg} /$ week and followed up for an average of 3.2 years. The primary end point was comparison of timeto-first MACE. The trial was powered to exclude a 1.8 or higher relative hazard of MACE for tocilizumab versus etanercept.

Results. By week 4, serum low-density lipoprotein cholesterol, high-density lipoprotein cholesterol, and triglyceride levels were $11.1 \%, 5.7 \%$, and $13.6 \%$ higher, respectively, for patients allocated to tocilizumab compared with etanercept (all $P<.001$ ). During follow-up, 83 MACE occurred in the tocilizumab group compared with 78 in the etanercept group. The estimated hazard of MACE for tocilizumab relative to etanercept was 1.05 (95\% confidence interval $=0.77,1.43)$. Result were similar in sensitivity analyses and the on-treatment analysis. Adverse events that occurred more frequently in the tocilizumab group included serious infection and gastrointestinal perforation.

Conclusion. The trial, which provides insights into the cardiovascular safety of tocilizumab versus etanercept, excluded a relative risk for MACE of 1.43 or higher. This result should be interpreted in the context of the clinical efficacy and the non-cardiovascular safety of tocilizumab. 
Keywords Biologicals, cardiovascular, randomized trial

Trial Registration. ClinicalTrials.gov identifier NCT01331837

Treatment with tocilizumab, a fully humanized monoclonal antibody targeting interleukin-6

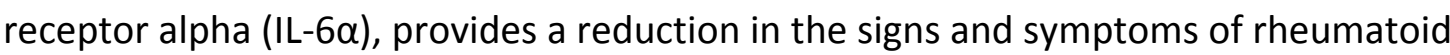
arthritis (RA) and a marked reduction in the levels of circulating inflammatory markers $(1,2)$. In parallel, treatment-associated increases in circulating lipid concentrations have been observed, with average increases in low-density lipoprotein cholesterol (LDL-C) of $12 \%$ to $20 \%$ noted across pivotal clinical trials (3). In a recent meta-analysis (4), the odds of having elevated LDL-C levels, defined as $>130 \mathrm{mg} / \mathrm{dL}$, was 4.8 -fold higher in tocilizumab-treated patients with RA than in patients treated with placebo. High-density lipoprotein cholesterol (HDL-C) also tended to increase with tocilizumab treatment because the odds of having high $\mathrm{HDL}-\mathrm{C}$, defined as $>60 \mathrm{mg} / \mathrm{dL}$, were more than 2-fold higher in tocilizumab-treated patients than in placebo-treated patients in the same meta-analysis. Considering that RA is associated with a greater burden of atherosclerosis $(5,6)$ and that deaths from atherosclerotic events and cardiovascular disease (CVD) are higher in patients with RA than in those without $(7,8)$, treatment-associated increased concentrations of lipids with atherogenic potential have called into question the CVD risk-to-benefit ratio of tocilizumab in RA.

In the MEASURE trial, designed to evaluate the effects of tocilizumab therapy on CVD biomarkers, concentrations of the more atherogenic circulating small LDL particles and oxidized LDL did not increase with tocilizumab treatment and were similar to those 
observed with placebo, despite an increase in overall LDL-C (9). At the same time, tocilizumab was associated with greater reductions, relative to placebo, in potentially proatherogenic factors, such as HDL-associated serum amyloid A, secretory phospholipase A2, and lipoprotein(a). However, whether such changes affect CVD event risk has not been evaluated in a clinical trial.

Given the increased levels of circulating total cholesterol, LDL-C, and triglycerides caused by tocilizumab, the ENTRACTE trial was designed to compare the risk for major adverse cardiovascular events (MACE) in patients with RA treated with tocilizumab and the risk in those treated with the tumor necrosis factor (TNF) inhibitor etanercept, a biologic standardof-care treatment for RA with a mechanism of action different from that of tocilizumab that has minimal effects on atherogenic lipids. Patients with high RA disease activity and baseline CVD risk factors were targeted.

\section{Methods}

Trial oversight and design. ENTRACTE (ClinicalTrials.gov number, NCT01331837) was a 2arm, randomized, open-label, parallel-group, multicenter trial designed to address the cardiovascular (CV) safety of tocilizumab versus that of etanercept in patients with RA. The trial was designed and executed as a postmarketing requirement of the US Food and Drug Administration. The sponsor was responsible for the study design, in collaboration with an external 6-member multidisciplinary steering committee who also supervised trial conduct; provided advice on scientific issues; was involved in the collection, analysis, and interpretation of data; wrote the manuscript; and approved the decision to submit the manuscript for publication. All authors were involved in analysis or interpretation of the 
data, and all authors made the decision to submit the manuscript for publication. The authors vouch for the accuracy and completeness of the data and for adherence to the trial protocol. The corresponding author had full access to all study data and had final responsibility for the decision to submit the paper for publication. The trial was approved by local institutional review boards/ethics committees. All patients provided written informed consent in accordance with the Declaration of Helsinki.

Patient population. Eligible patients had RA, based on the American College of Rheumatology 1987 criteria (10), of $\geq 6$ months' duration, inadequate response to a previous conventional synthetic disease-modifying antirheumatic drug (csDMARD) or anti-TNF treatment, seropositivity for rheumatoid factor (RF) or anti-cyclic citrullinated protein (antiCCP), $\geq 8$ swollen joints ( 66 joint count) and 8 tender joints ( 68 joint count) at screening, and C-reactive protein (CRP) $>0.3 \mathrm{mg} / \mathrm{dL}$. Additionally, patients were required to be $\geq 50$ years old and to have $\geq 1$ traditional CVD risk factors, extra-articular RA manifestations (including subcutaneous rheumatoid nodules, secondary Sjogren syndrome, serositis, rheumatoid lung disease/ interstitial lung disease, vasculitis, inflammatory peripheral neuropathy, or scleritis/ episcleritis), or history of a CVD event, which included prior MI, CVA, coronary revascularization procedure, hospitalization for unstable angina, symptomatic carotid artery disease, peripheral arterial disease, or abdominal aortic aneurysm. Patients with moderate or severe heart failure were excluded due to the contraindication of randomization to a TNF inhibitor. Those who previously received treatment with a non-TNF biologic or etanercept were excluded, and enrollment of those who previously received treatment with nonetanercept TNF inhibitors was restricted to $20 \%$. Individuals with a history of diverticulitis, diverticulosis requiring antibiotic treatment or chronic ulcerative lower gastrointestinal 
disease such as Crohn's disease, ulcerative colitis or other symptomatic lower gastrointestinal conditions that might predispose to perforations were excluded. Additional inclusion and exclusion criteria are included in the Supplementary Appendix.

Intervention, randomization, and blinding. Patients were randomly assigned using an interactive voice recording system (IVRS) 1:1 to either intravenous tocilizumab ( $8 \mathrm{mg} / \mathrm{kg}$ every 4 weeks) or subcutaneous etanercept (50 mg weekly), with or without background $\operatorname{csDMARD(s).~A~pre-allocated~blocked~randomization~schedule~was~implemented~using~a~}$ block size of 4 and was stratified according to previous exposure to anti-TNF therapy and history of CVD events. The random allocation sequence was generated by an independent vendor who managed the IVRS. Patients were enrolled by study site personnel and randomly assigned using the IVRS. Tocilizumab was administered at the study site, whereas etanercept was self-administered at home. The trial was open label at the study site but blinded to the sponsor. Site monitoring was independent of the sponsor, and data on treatment allocation was not divulged to the sponsor by monitors. An independent data monitoring committee reviewed unblinded data and monitored safety.

Study visits. On-site study visits occurred monthly for tocilizumab-treated patients and every 3 months for etanercept-treated patients during years 1-3 and every 6 months during years 4-5. Enrollees could withdraw from active treatment at any time; crossover was discouraged, however, and patients who withdrew from treatment were encouraged to continue to be monitored for ongoing CVD and safety assessments. The trial commenced on August 2, 2011, and concluded on March 25, 2016.

This article is protected by copyright. All rights reserved. 
End points and safety assessments. The prespecified primary end point was time to first occurrence of MACE, consisting of CV death, nonfatal myocardial infarction (MI), and nonfatal stroke (of any type). Undetermined causes of death were classified as MACE according to the Standardized Definitions of End Points in Clinical Trials (11). A secondary end point was time to first occurrence of an expanded composite end point, consisting of MACE + non-elective coronary revascularization procedures and hospitalization for unstable angina. Other secondary end points were all-cause mortality and time to first occurrence of each of the individual components of MACE. Exploratory end points included hospitalized heart failure (HHF) and MACE + HHF.

CVD events and serious adverse events (SAEs) were assessed monthly throughout the trial, either on site or through a telephone call using an interactive voice response system. Any affirmative responses resulted in an alert for the site to contact the patient for additional detail on the possible adverse event (AE) or SAE. All potential end point events were adjudicated by a blinded independent CVD Events Adjudication Committee. In the circumstance that the event was adjudicated as not a confirmed event, the patient was still considered at risk for a first event. No efficacy data were assessed.

Laboratory assessments. Peripheral blood was collected at 1 month and 3 months following randomization, every 3 months until year 3, and every 6 months from year 4 onward. Nonfasting total cholesterol, triglyceride, HDL-C, and LDL-C levels were measured by Roche (Basel, Switzerland)-BMD methodology, RF was measured by Roche Tina-quant immunoturbidimetric assay, anti-CCP was measured by enzyme-linked immunosorbent 
assay, hemoglobin A1C was measured by Bio-Rad (Hercules, CA) high-performance liquid chromatography, and CRP was measured by Roche Tina-quant immunoturbidimetric assay.

Statistical analysis. The primary objective of this trial was to provide a screening evaluation of cardiovascular safety by addressing whether a hazard ratio (HR) of 1.8 for MACE could be ruled out in the comparison between tocilizumab and etanercept. This would be achieved in a 131-event trial by an estimated $H R \leq 1.278$, which would occur with $92 \%$ probability if the true HR were 1 . The primary analysis of the MACE primary end point and of all secondary and exploratory time-to-event end points was based on a stratified Cox proportional hazards model using the intention-to-treat (ITT) population. Treatment arm was included as a covariate in the model, and the analysis was stratified by previous exposure to anti-TNF therapy and history of CVD events. For the "on treatment" analyses, exposure time was truncated at 28 days after the last dose of randomized treatment unless a new biologic was started during that period. In this case, exposure time was truncated at the date of the initiation of the new biologic.

In many previous CV safety trials conducted in settings such as the evaluation of antidiabetes agents, obesity therapies, and cyclooxygenase- 2 inhibitors and other nonsteroidal anti-inflammatory drugs in osteoarthritis and RA, a definitive evaluation of CV safety typically has required ruling out a noninferiority margin for the HR of MACE in the range of 1.30 to 1.33 . In those settings, ruling out much larger increases in the range of 1.8 to 2.0 has provided a screening evaluation for CV safety $(12,13)$. The design of ENTRACTE was consistent with this screening evaluation. Its primary objective, as stated, was to address whether a possible true HR of 1.8 could be ruled out (achieved when the upper limit of the 
$95 \% \mathrm{Cl}<1.8$ ). If that were to be achieved, then, in a nested manner preserving the experimental alpha level of a traditional $2.5 \%$ false positive error rate, a conclusion of "noninferiority of tocilizumab to etanercept" could be formally established if a possible true HR of 1.3 could be ruled out (achieved when the upper limit of the $95 \% \mathrm{Cl}<1.3$ ), where this would be achieved in a 131-event trial by an estimated HR $\leq 0.923$ (or in a 161-event trial by an estimated $H R \leq 0.954)$. The corresponding sample size was $n=1540$ per treatment arm. Non-CVD safety events were assessed in the on-treatment safety population.

\section{Results}

A total of 3080 patients with RA were enrolled; 1538 were randomly assigned to the tocilizumab arm and 1542 were randomly assigned to the etanercept arm. The tocilizumab and etanercept arms contributed 4900 and 4891 ITT patient-years, respectively. The disposition of patients through the trial is depicted in Figure 1. Over an average follow-up time of 3.2 years, 2957 patients (96\%) completed the trial with a full assessment of CVD events. Excluding those who died during follow-up, there were 26 patients in the tocilizumab group and 33 patients in the etanercept group without a full assessment of CVD events. Early discontinuation of randomized treatment occurred in 361 of 1542 (23\%) etanercept-treated patients and 401 of 1538 (26\%) tocilizumab-treated patients, representing 518.5 and 655.2 patient-years, respectively. Etanercept-to-tocilizumab and tocilizumab-to-etanercept crossover occurred equally in $1 \%$ of enrollees in each arm.

Baseline characteristics are summarized in Table 1 according to randomized treatment assignment. Characteristics were balanced across the treatment arms and reflected a population selected for having severe/active RA and being at high risk for CVD events. 
Following initiation of study treatment, the tocilizumab group had a median $11.1 \%$ greater increase in LDL-C level compared with the etanercept group, 5.4\% greater increase in HDL-C level, and $13.6 \%$ greater increase in triglyceride level at 4 weeks (all $P<.001$ ). These effects persisted over time (Figure 2).

One hundred sixty-one patients experienced primary MACE events, 78 in the etanercept arm and 83 in the tocilizumab arm. Although the trial was designed for 131 events, 30 additional events accrued because of inherent imprecision in identifying the proper timing of the data lock; this was further complicated by the lag time between identification and adjudication of possible events. In a comparison of tocilizumab with etanercept, the ITT HR for the primary end point of MACE that included undetermined cause of death was 1.05 (95\% confidence interval [Cl], 0.77-1.43) (Table 2) (Kaplan-Meier plots for the primary end point are included in Supplementary Figure S1 in the Supplementary Appendix). The estimated HR was similar in sensitivity analyses using alternative classifications of MACE and in the analysis restricted to the period when patients were actually receiving the treatment to which they were assigned. The estimated HR for tocilizumab versus etanercept for the secondary end points provided in Table 2 ranged from 0.89 for non-fatal $\mathrm{MI}(95 \% \mathrm{Cl} 0.54-$ 1.49) to 1.55 for all stroke types ( $95 \% \mathrm{Cl} 0.83-2.90)$. For fatal and nonfatal stroke, 26 events occurred in the tocilizumab group compared with 16 events in the etanercept group. The HRs for cardiovascular death and death from any cause for the tocilizumab versus etanercept groups were 1.03 and 0.99 , respectively.

This article is protected by copyright. All rights reserved. 
Non-CVD AEs were common in both groups, but the rate was higher in the tocilizumab arm than in the etanercept arm (Table 3). However, neither AEs leading to discontinuation of study drug nor SAEs were meaningfully higher in the tocilizumab arm than in the etanercept arm. Infections (any infections and serious infections) were higher in the tocilizumab arm than in the etanercept arm. Eight confirmed gastrointestinal perforation events occurred in the tocilizumab arm compared with 1 event in the etanercept arm. Rates of malignancy, hypersensitivity events, serious bleeding events, and serious hepatic events were not meaningfully higher in the tocilizumab arm than in the etanercept arm, and no demyelinating events occurred.

\section{Discussion}

The primary aim of the ENTRACTE trial was to determine whether a relative risk for MACE of 1.8 or higher for tocilizumab versus etanercept could be ruled out. The trial, which provides screening insights into the CVD safety of tocilizumab compared with etanercept, excluded a relative risk for MACE of 1.43 or higher in the ITT population. Similar estimates regarding CVD safety were obtained when the analysis was restricted to events that occurred while patients were still receiving randomized treatment and regarding multiple secondary and exploratory outcomes, with the exception of stroke and HHF.

Macrophage-derived inflammatory cytokines, including IL-6, TNF- $\alpha$, and IL-1 $\beta$, are upregulated in RA and are targets for effective treatment of the signs and symptoms of the disease (14). Multiple studies support a role for IL-6 as a key contributor to atherogenesis, plaque destabilization, and atherothrombosis (15-18). Similar to IL-6 levels, higher TNF- $\alpha$ levels have been linked to future CVD events (19), and TNF knockout or treatment with TNF 
inhibitors was associated with a reduction in the burden of atherosclerosis in atherosclerosis-prone mice $(20,21)$. Moreover, a growing body of evidence from observational registries of TNF inhibitor-treated patients with RA suggests that CVD events are lower with TNF inhibitor treatment (22), an effect that may depend on clinical response (23). However, whether this effect is unique to TNF inhibitors as a class or extends to targeted immunomodulators with different mechanisms of action is unknown. In a recent analysis of Medicare and insurance claims beneficiaries(24), Xie et al reported a relative hazard of MACE for etanercept vs. tocilizumab of $1.10(95 \% \mathrm{Cl}=0.80,1.51)$. In a separate analysis of Medicare and private insurance claims (25), Kim et al also reported a lower but non-significant hazard of hospitalization for myocardial infarction or stroke for those prescribed tocilizumab compared to a combined TNF inhibitor group $[\mathrm{HR}=0.84(95 \% \mathrm{Cl}=0.56$, 1.26)]. However, given the non-randomized treatment allocation and retrospective design, a randomized clinical trial, such as ENTRACTE, is needed to more accurately estimate the true relative risk between the treatments. Additionally, the concept that cytokine inhibition may have antiatherogenic or antithrombotic effects, or both, on conditions other than RA is supported by the recently published Canakinumab Anti-inflammatory Thrombosis Outcomes Study, in which anti-IL-1 $\beta$ treatment of patients who previously experienced MI and elevated CRP levels was associated with a $15 \%$ reduction, compared with placebo, in the risk for MACE over 48 months (26).

On a molecular level, IL-6 inhibition differs from TNF inhibition in several pathways involved in lipid metabolism. RA is associated with LDL hypercatabolism, an effect that in 1 study diminished with tocilizumab treatment, resulting in higher LDL-C levels (27). Additionally, tocilizumab was found to decrease IL-6-induced expression of the LDL receptor 
(LDLr) on cultured hepatocytes, an effect not observed with the TNF inhibitor adalimumab (28). Such downregulation of LDLr on hepatocytes and other LDLr-expressing cells would be expected to result in higher circulating LDL levels. In the same study, tocilizumab treatment was associated with higher postprandial triglyceride levels and a delayed triglyceride peak after oral fat loading, suggesting attenuation of IL-6-induced lipolysis (28). The exact mechanism of tocilizumab-associated increases in levels of circulating triglycerides, however, is unclear. Perhaps more important is whether these tocilizumab-associated changes in lipid metabolism translate to actual CVD risk over time. Based on the findings of ENTRACTE, CVD risk on tocilizumab does not appear to be markedly increased compared with that on etanercept, at least within the first several years after therapy initiation, yet we cannot rule out a difference in relative hazard smaller than 1.43 given the width of the resulting $\mathrm{Cl}$ for MACE. The certainty around the relative differences between tocilizumab and etanercept for the secondary and exploratory outcomes, particularly for stroke and $\mathrm{HHF}$, was lower, largely driven by the overall infrequency of these outcomes. Given this infrequency, an even larger trial than ENTRACTE would be needed to stringently explore comparative differences on any one of the secondary outcomes.

ENTRACTE has a number of notable strengths and limitations. Among the strengths, the retention rate for assessment of CVD events was very high (96\%), and the unintended crossover rate was low (1\%), both of which are indicators of a well-executed safety trial designed to assess whether differences could be ruled out. The use of an external blinded CVD events adjudication committee eliminated the possibility of bias in the attribution of CVD events, and an automated call-in reporting system reduced the likelihood of reporting bias for CVD events and SAEs. However, more face-to-face opportunities for tocilizumab- 
treated patients to report AEs directly to study personnel provided an imbalanced sensitivity to detecting AEs between arms, which might have contributed to more nonserious safety events being reported for patients receiving tocilizumab therapy. The tocilizumab group experienced a higher infection rate and a significantly higher rate of gastrointestinal perforations. Although both these rates were consistent with rates observed in previous tocilizumab clinical trials (29), they do provide additional insight into the comparative safety of tocilizumab for RA therapy relative to etanercept.

The limitations of this study include the fact that although the sample size of ENTRACTE was larger than in a typical RA trial with a clinical efficacy end point, it was relatively small for a trial with a CVD event end point, in part because the eligible RA population is much smaller than the diabetes and obesity populations. As such, though it is estimated that the risk for MACE in patients treated with tocilizumab is $5 \%$ higher than in patients treated with etanercept, the uncertainty around this estimate was wide enough that the true risk for MACE in the tocilizumab arm could have been anywhere from $43 \%$ higher to $23 \%$ lower than in the etanercept arm. Although the trial did not rule out traditional noninferiority margins in the range of 1.30 to 1.33 , it did succeed in ruling out the larger 1.8 relative risk for MACE in patients receiving tocilizumab compared with those receiving etanercept, as a CV safety screening trial, that it was designed to address. Finally, ENTRACTE enrolled an RA population remarkable for having both high RA disease activity and elevated CVD risk, which may not reflect the characteristics of the average patient with RA in clinical practice. Nevertheless, there is biologic reason to believe that the CVD safety of tocilizumab compared with that of etanercept can be extrapolated to patients with RA who have lower baseline disease activity or lower CVD risk.

This article is protected by copyright. All rights reserved. 
Additionally, while the drop-out rate for the trial was very low (4\%), this rate was similar to the overall MACE rate. Thus, while unlikely, there is potential for even this small amount of drop-out to bias the comparison of MACE between the two groups. Finally, since the trial was powered on CV safety, measures of RA disease activity were not collected after baseline. Powering the trial on both CV safety and clinical efficacy would have required the enrollment of a much larger sample size than what was feasible.

In summary, the primary results of the ENTRACTE trial provide a framework for assessing the comparative CVD safety of tocilizumab with that of etanercept in the setting of treatment-associated increases in lipid parameters and in the context of its known clinical efficacy and non-CVD safety.

\section{AUTHOR CONTRIBUTIONS}

All authors were involved in the analysis or interpretation of the data, and all authors made the decision to submit the manuscript for publication. The authors vouch for the accuracy and completeness of the data and for adherence to the trial protocol, available with the full text of this article. The trial was approved by local institutional review boards/ethics committees.

Jon T. Giles: 1c, 2, 3.

Naveed Sattar: 1c, 2, 3.

Sherine Gabriel: 1c, 2, 3.

Paul M. Ridker: 1c, 2, 3.

Steffen Gay: 1c, 2, 3.

This article is protected by copyright. All rights reserved. 
Charles Warne: 1c, 2, 3.

David Musselman: 1c, 2, 3.

Laura Brockwell: 1c, 2, 3.

Emma Shittu: 1c, 2, 3.

Micki Klearman: 1c, 2, 3.

Thomas R. Flemming: 1c, 2, 3.

\section{ROLE OF THE STUDY SPONSOR}

This study was funded by F. Hoffmann-La Roche Ltd. (Roche). Roche was involved in the design and conduct of the study; collection, management, analysis, and interpretation of the data; and preparation, review, and approval to submit the manuscript for publication.

\section{DATA SHARING STATEMENT}

Qualified researchers may request access to data through the clinical study data request platform (www.clinicalstudydatarequest.com). Further details on Roche's criteria for eligible studies are available here (https://clinicalstudydatarequest.com/Study-Sponsors/StudySponsors-Roche.aspx). For further details on Roche's Global Policy on the Sharing of Clinical Information and how to request access to related clinical study documents, see here (https://www.roche.com/research_and_development/who_we_are_how_we_work/clinical _trials/our_commitment_to_data_sharing.htm)

This article is protected by copyright. All rights reserved. 


\section{REFERENCES}

1. Genovese MC, McKay JD, Nasonov EL, Mysler EF, da Silva NA, Alecock E, et al. Interleukin6 receptor inhibition with tocilizumab reduces disease activity in rheumatoid arthritis with inadequate response to disease-modifying antirheumatic drugs: the tocilizumab in combination with traditional disease-modifying antirheumatic drug therapy study. Arthritis Rheum 2008;58:2968-2980.

2. Smolen JS, Beaulieu A, Rubbert-Roth A, Ramos-Remus C, Rovensky J, Alecock E, et al. Effect of interleukin-6 receptor inhibition with tocilizumab in patients with rheumatoid arthritis (OPTION study): a double-blind, placebo-controlled, randomised trial. Lancet 2008;371:987-997.

3. Schiff MH, Kremer JM, Jahreis A, Vernon E, Isaacs JD, van Vollenhoven RF. Integrated safety in tocilizumab clinical trials. Arthritis Res Ther 2011;13:R141.

4. Souto A, Salgado E, Maneiro JR, Mera A, Carmona L, Gomez-Reino JJ. Lipid profile changes in patients with chronic inflammatory arthritis treated with biologic agents and tofacitinib in randomized clinical trials: a systematic review and meta-analysis. Arthritis Rheumatol 2015;67:117-127.

5. Giles JT, Szklo M, Post W, Petri M, Blumenthal RS, Lam G, et al. Coronary arterial calcification in rheumatoid arthritis: comparison to the multi-ethnic study of atherosclerosis. Arthritis research \& therapy 2009;11:R36.

6. Karpouzas GA, Malpeso J, Choi TY, Li D, Munoz S, Budoff MJ. Prevalence, extent and composition of coronary plaque in patients with rheumatoid arthritis without symptoms or prior diagnosis of coronary artery disease. Ann Rheum Dis 2014;73:1797-1804.

7. Avina-Zubieta JA, Choi HK, Sadatsafavi M, Etminan M, Esdaile JM, Lacaille D. Risk of cardiovascular mortality in patients with rheumatoid arthritis: a meta-analysis of observational studies. Arthritis and Rheumatism 2008;59:1690-1697.

8. Avina-Zubieta JA, Thomas J, Sadatsafavi M, Lehman AJ, Lacaille D. Risk of incident cardiovascular events in patients with rheumatoid arthritis: a meta-analysis of observational studies. Ann Rheum Dis 2012;71:1524-1529.

9. McInnes IB, Thompson L, Giles JT, Bathon JM, Salmon JE, Beaulieu AD, et al. Effect of interleukin- 6 receptor blockade on surrogates of vascular risk in rheumatoid arthritis: MEASURE, a randomised, placebo-controlled study. Ann Rheum Dis 2015;74:694-702.

10. Arnett FC, Edworthy SM, Bloch DA, McShane DJ, Fries JF, Cooper NS, et al. The American Rheumatism Association 1987 revised criteria for the classification of rheumatoid arthritis. Arthritis and Rheumatism 1988;31:315-324.

11. Hicks KA, Tcheng JE, Bozkurt B, Chaitman BR, Cutlip DE, Farb A, et al. 2014 ACC/AHA

Key Data Elements and Definitions for Cardiovascular Endpoint Events in Clinical Trials: A

This article is protected by copyright. All rights reserved. 
Report of the American College of Cardiology/American Heart Association Task Force on Clinical Data Standards (Writing Committee to Develop Cardiovascular Endpoints Data Standards). Circulation 2015;132:302-361.

12. U.S. Food and Drug Administration. Guidance for Industry. Diabetes MellitusEvaluating Cardiovascular Risk in New Antidiabetic Therapies to Treat Type 2 Diabetes December 2008 [cited 2018 July 1]. Available from:

www.fda.gov/downloads/Drugs/GuidanceComplianceRegulatoryInformation/Guidances/uc m071627.pdf.

13. Fleming TR. Identifying and addressing safety signals in clinical trials: Some Issues and Challenges. Proceedings of the 4th Seattle Symposium in Biostatistics: Clinical Trials 2013. p. 137-156.

14. Li J, Hsu HC, Mountz JD. Managing macrophages in rheumatoid arthritis by reform or removal. Curr Rheumatol Rep 2012;14:445-454.

15. Kaptoge S, Seshasai SR, Gao P, Freitag DF, Butterworth AS, Borglykke A, et al. Inflammatory cytokines and risk of coronary heart disease: new prospective study and updated meta-analysis. Eur Heart J 2014;35:578-589.

16. Interleukin-6 Receptor Mendelian Randomisation Analysis C, Swerdlow DI, Holmes MV, Kuchenbaecker KB, Engmann JE, Shah T, et al. The interleukin-6 receptor as a target for prevention of coronary heart disease: a mendelian randomisation analysis. Lancet 2012;379:1214-1224.

17. Collaboration IRGCERF, Sarwar N, Butterworth AS, Freitag DF, Gregson J, Willeit P, et al. Interleukin- 6 receptor pathways in coronary heart disease: a collaborative meta-analysis of 82 studies. Lancet 2012;379:1205-1213.

18. Maier W, Altwegg LA, Corti R, Gay S, Hersberger M, Maly FE, et al. Inflammatory markers at the site of ruptured plaque in acute myocardial infarction: locally increased interleukin- 6 and serum amyloid $A$ but decreased C-reactive protein. Circulation 2005;111:1355-1361.

19. Cesari M, Penninx BW, Newman AB, Kritchevsky SB, Nicklas BJ, Sutton-Tyrrell K, et al. Inflammatory markers and onset of cardiovascular events: results from the Health $A B C$ study. Circulation 2003;108:2317-2322.

20. Ohta $\mathrm{H}$, Wada $\mathrm{H}$, Niwa $\mathrm{T}$, Kirii $\mathrm{H}$, Iwamoto $\mathrm{N}$, Fujii $\mathrm{H}$, et al. Disruption of tumor necrosis factor-alpha gene diminishes the development of atherosclerosis in ApoE-deficient mice. Atherosclerosis 2005;180:11-17.

21. Branen L, Hovgaard L, Nitulescu M, Bengtsson E, Nilsson J, Jovinge S. Inhibition of tumor necrosis factor-alpha reduces atherosclerosis in apolipoprotein $\mathrm{E}$ knockout mice. Arteriosclerosis, Thrombosis, and Vascular Biology 2004;24:2137-2142.

22. Roubille C, Richer V, Starnino T, McCourt C, McFarlane A, Fleming P, et al. The effects of tumour necrosis factor inhibitors, methotrexate, non-steroidal anti-inflammatory drugs

This article is protected by copyright. All rights reserved. 
and corticosteroids on cardiovascular events in rheumatoid arthritis, psoriasis and psoriatic arthritis: a systematic review and meta-analysis. Ann Rheum Dis 2015;74:480-489.

23. Dixon WG, Watson KD, Lunt M, Hyrich KL, British Society for Rheumatology Biologics Register Control Centre C, Silman AJ, et al. Reduction in the incidence of myocardial infarction in patients with rheumatoid arthritis who respond to anti-tumor necrosis factor alpha therapy: results from the British Society for Rheumatology Biologics Register. Arthritis Rheum 2007;56:2905-2912.

24. Xie F, Yun H, Levitan EB, Muntner P, Curtis JR. Tocilizumab and the risk for cardiovascular disease: a direct comparison among biologic disease-modifying antirheumatic drugs for rheumatoid arthritis patients. Arthritis Care Res (Hoboken) 2018.

25. Kim SC, Solomon DH, Rogers JR, Gale S, Klearman M, Sarsour K, et al. Cardiovascular Safety of Tocilizumab Versus Tumor Necrosis Factor Inhibitors in Patients With Rheumatoid Arthritis: A Multi-Database Cohort Study. Arthritis Rheumatol 2017;69:1154-1164.

26. Ridker PM, Everett BM, Thuren T, MacFadyen JG, Chang WH, Ballantyne C, et al. Antiinflammatory Therapy with Canakinumab for Atherosclerotic Disease. N Engl J Med 2017;377:1119-1131.

27. Robertson J, Porter D, Sattar N, Packard CJ, Caslake M, Mclnnes I, et al. Interleukin-6 blockade raises LDL via reduced catabolism rather than via increased synthesis: a cytokinespecific mechanism for cholesterol changes in rheumatoid arthritis. Ann Rheum Dis 2017;76:1949-1952.

28. Strang AC, Bisoendial RJ, Kootte RS, Schulte DM, Dallinga-Thie GM, Levels JH, et al. Pro-atherogenic lipid changes and decreased hepatic LDL receptor expression by tocilizumab in rheumatoid arthritis. Atherosclerosis 2013;229:174-181.

29. Xie F, Yun H, Bernatsky S, Curtis JR. Brief Report: Risk of Gastrointestinal Perforation Among Rheumatoid Arthritis Patients Receiving Tofacitinib, Tocilizumab, or Other Biologic Treatments. Arthritis Rheumatol 2016;68:2612-2617.

This article is protected by copyright. All rights reserved. 


\section{FIGURE LEGENDS}

Figure 1. Disposition of ENTRACTE trial participants. The primary end point was time to first CV-EAC adjudicated event (CV death, nonfatal MI, or nonfatal stroke) in the ITT population. Treatment completers are defined as patients who were not marked as discontinuing from randomized treatment before the CSED 30-/90-day window. The early discontinuation from randomized treatment count does not include patients who were incorrectly marked as discontinuing treatment within the CSED 3-0/90-day window (10 patients). Reasons for inclusion in the "Miscellaneous" groups were the following: lost to follow-up, protocol violation, noncompliance with study drug, and noncompliance with protocol. Study completers were defined as patients who experienced a primary end point or completed their final study contact by direct telephone contact or site visit within the CSED 30-/90-day window. Only patients who died of non-CV-related causes or who died of CV-related causes $>365$ days after the last direct contact (therefore not classified as a primary end point) are reported as having discontinued from the study because of death. CSED = common study end date; CV = cardiovascular; EAC = cardiovascular events adjudication committee; ETA = etanercept; $\mathrm{MI}=$ myocardial infarction; $\mathrm{TCZ}=$ tocilizumab.

Figure 2. Baseline and change in circulating lipoprotein and triglyceride levels over 216 weeks: etanercept versus tocilizumab. Medians and interquartile ranges are depicted. ETA = etanercept; HDL = high-density lipoprotein; IQR = interquartile range; IV = intravenous; LDL = low-density lipoprotein; qw = every week; q4w = every 4 weeks; SC = subcutaneous; TCZ = tocilizumab.

This article is protected by copyright. All rights reserved. 
Table 1. Baseline Characteristics According to Randomized Treatment Allocation (intention-to-treat population)

Baseline Characteristics

Male sex, $\mathrm{n}(\%)$

White race, $\mathrm{n}(\%)$

Body mass index, $\mathrm{kg} / \mathrm{m}^{2}$ (mean $\left.\pm \mathrm{SD}\right)$

CVD risk factors

Current smoking, $\mathrm{n}(\%)$

Hypertension, $\mathrm{n}(\%)$

Diabetes, $\mathrm{n}(\%)$

Family history of CVD, $\mathrm{n}(\%)$

Total cholesterol, mg/dL (mean \pm SD)

$\mathrm{LDL}-\mathrm{C}, \mathrm{mg} / \mathrm{dL}($ mean $\pm \mathrm{SD})$

$\mathrm{HDL}-\mathrm{C}, \mathrm{mg} / \mathrm{dL}($ mean $\pm \mathrm{SD})$

Triglycerides, $\mathrm{mg} / \mathrm{dL}$ (interquartile range)

Hemoglobin A1C, \% (mean \pm SD)

Current statin use, $\mathrm{n}(\%)$

Previous CVD diagnoses, events, and procedures

Any previous CVD diagnoses, events, or procedures, $\mathrm{n}$

(\%)

$\mathrm{MI}, \mathrm{n}(\%)$

Stroke, n (\%)

Coronary revascularization, $\mathrm{n}(\%)$
Etanercept

$n=1542$

$61 \pm 8$

$340(22)$

$1187(77)$

$29.0 \pm 6.0$

$28.7 \pm 5.9$

$419(27)$

$1090(71)$

$284(18)$

239 (16)

$198 \pm 42$

$113 \pm 35$

$57 \pm 16$

123 (92-168)

$5.9 \pm 1.1$

$5.9 \pm 1.1$

$343(22)$

$333(22)$

184 (11.9)

$163(10.6)$

79 (5)

$67(4)$

$35(2)$

$39(3)$

72 (5)

$54(4)$

This article is protected by copyright. All rights reserved. 
Hospitalized for unstable angina, n (\%)

Symptomatic carotid artery disease, $\mathrm{n}(\%)$

Peripheral arterial disease, $\mathrm{n}(\%)$

RA characteristics

Duration, years (interquartile range)

Rheumatoid factor seropositive, $\mathrm{n}(\%)$

Anti-CCP seropositive, $\mathrm{n}(\%)$

$\mathrm{CRP}, \mathrm{mg} / \mathrm{L}$ (interquartile range)

Any extra-articular RA features, n (\%)

Prior Tumor Necrosis Inhibitor Use, n (\%)

Concurrent RA therapies

Methotrexate, n (\%)

Antimalarials, $\mathrm{n}(\%)$

Sulfasalazine, $\mathrm{n}(\%)$

Leflunomide, $\mathrm{n}(\%)$

Corticosteroids, n (\%)

NSAIDs, $\mathrm{n}(\%)$
$44(3)$

$50(3)$

$14(1)$

$7(1)$

$29(2)$

15 (1)

$\begin{array}{cc}7.2(3.1-4.6) & 7.9(3.1-14.7) \\ 1485(97) & 1475(97) \\ 1139(90) & 1149(91) \\ 10.5(4.9-23.4) & 10.4(4.8-23.0) \\ 465(30) & 462(30) \\ 41(2.7 \%) & 33(2.1 \%)\end{array}$

$1098(71)$

1111 (72)

$193(13)$

$213(14)$

$153(10)$

167 (11)

168 (11)

161 (11)

400 (26)

445 (29)

$814(53)$

$828(54)$

$\mathrm{CCP}=$ cyclic citrullinated protein; $\mathrm{CRP}=\mathrm{C}$-reactive protein; $\mathrm{CVD}=$ cardiovascular disease; $\mathrm{HDL}-\mathrm{C}=$ high-density lipoprotein C; LDL-C = low-density lipoprotein C; MI = myocardial infarction; NSAIDs = nonsteroidal anti-inflammatory drugs; RA = rheumatoid arthritis; SD = standard deviation.

This article is protected by copyright. All rights reserved. 
Table 2. Number of first occurrence events and relative hazards (tocilizumab versus etanercept) for primary and secondary end points, exploratory end points,

and sensitivity analyses

End Point

First Events

$\begin{array}{llll}\text { Etanercept } & \text { a } & \text { Tocilizumab }^{\mathrm{a}} & \mathrm{HR}^{\mathrm{b}} \quad 95 \% \mathrm{Cl}^{\mathrm{f}}\end{array}$

$n(\%) \quad$ events $/ 100$ pys $(95 \% \mathrm{Cl}) \quad n(\%) \quad$ events/100 pys $(95 \% \mathrm{Cl})$

Primary end point: intention-to-treat population ${ }^{c}$

MACE, including undetermined cause of death, $n(\%)$

Primary end point: on-treatment population ${ }^{\mathrm{d}}$

MACE, including undetermined cause of death, $n(\%)$

$78(5)$

$1.70(1.35,2.10)$

$83(5) \quad 1.82(1.46,2.24)$

1.05

$0.77-1.43$

Sensitivity analysis of primary end point:

$52(3) \quad 1.28(0.97,1.66)$

$57(4) \quad 1.44(1.10,1.85)$

$1.11 \quad 0.76-1.62$

intention-to-treat population

MACE, excluding undetermined cause of death, $n(\%)$

$72(5) \quad 1.57(1.24,1.97)$

$74(5) \quad 1.63(1.29,2.03)$

$1.01 \quad 0.73-1.40$

MACE, before last direct contact, $n(\%)$

$46(3) \quad 1.00(0.74,1.33)$

$49(3) \quad 1.06(0.79,1.40)$

$1.04 \quad 0.70-1.56$

Secondary end points:

intention-to-treat population

Nonfatal MI, $\mathrm{n}(\%)$

Nonfatal and fatal $\mathrm{MI}, \mathrm{n}(\%)$

Nonfatal stroke, all types, $\mathrm{n}(\%)$

Nonfatal and fatal stroke, all types, $\mathrm{n}(\%)$

Cardiovascular death, $\mathrm{n}(\%)$

Death from any cause, $\mathrm{n}(\%)$

Expanded composite end point, ${ }^{e} \mathrm{n}(\%)$

Exploratory end points: intention-to-treat population

MACE + hospitalized heart failure, $n(\%)$

$31(2) \quad 0.65(0.45,0.92)$

$32(2) \quad 0.67(0.46,0.95)$

$28(2) \quad 0.59(0.40,0.85)$

$29(2) \quad 0.61(0.41,0.87)$

$15(1) \quad 0.33(0.19,0.53)$

$24(2) \quad 0.49(0.31,0.73)$

$16(1) \quad 0.35(0.20,0.56)$

$35(2) \quad 0.72(0.50,1.00)$

$26(2) \quad 0.53(0.35,0.78)$

$64(4) \quad 1.31(1.01,1.67)$

$36(2) \quad 0.73(0.51,1.02)$

$64(4) \quad 1.31(1.01,1.67)$

$84(6) \quad 1.90(1.53,2.33)$

$0.89 \quad 0.54-1.49$

$0.90 \quad 0.54-1.48$

$\begin{array}{ll}1.53 & 0.80-2.92\end{array}$

$\begin{array}{ll}1.55 & 0.83-2.90\end{array}$

$84(5) \quad 1.98(1.61,2.42)$

90 (6) $2.12(1.73,2.57)$

$85(6) \quad 1.90(1.53,2.33)$

12 (1) $\quad 0.31(0.17,0.50)$

$1.03 \quad 0.64-1.63$

$0.99 \quad 0.70-1.41$

$0.99 \quad 0.73-1.34$

8 (1) $\quad 0.20(0.10,0.38)$

$1.05 \quad 0.78-1.41$

$1.50 \quad 0.61-3.67$

This article is protected by copyright. All rights reserved. 
${ }^{a}$ Etanercept $50 \mathrm{mg}$ subcutaneous injection weekly; tocilizumab $8 \mathrm{mg} / \mathrm{kg}$ intravenous infusion monthly.

${ }^{\mathrm{b}}$ The hazard ratio was estimated by Cox regression including treatment (tocilizumab/etanercept) as the only covariate, stratified by previous exposure to anti-TNF therapy and history of CV events. Time to event was calculated from (randomization to first event or censoring date +1 )/365.25.

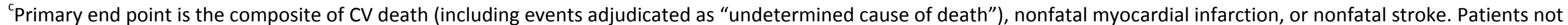
experiencing an event were censored at the latest of last date known to be alive, last direct contact, and last indirect contact, capped at the date of last direct contact +365 days.

${ }^{d}$ Censoring date is the latest of last date known to be alive, last direct contact, and last indirect contact, capped at the date of randomized treatment discontinuation or the common study end date, whichever is earlier.

${ }^{e}$ Defined as the $\mathrm{CV}$ composite of the primary end point, with the addition of nonelective coronary revascularization procedures and hospitalization for unstable angina.

${ }^{f}$ The confidence intervals for secondary end points should be viewed only in a descriptive manner because they have not been adjusted for multiplicity.

$\mathrm{Cl}=$ confidence interval; $\mathrm{CV}=$ cardiovascular; $\mathrm{HR}=$ hazard ratio; $\mathrm{MACE}=$ major adverse cardiovascular event $\mathrm{MI}=$ myocardial infarction; $\mathrm{TNF}=$ tumor necrosis factor.

This article is protected by copyright. All rights reserved. 
Table 3. Adverse Events: On-Treatment Safety Population

\begin{tabular}{|c|c|c|c|c|c|c|c|c|}
\hline \multirow[t]{2}{*}{ Adverse Events } & \multicolumn{3}{|c|}{ Etanercept } & \multicolumn{3}{|c|}{ Tocilizumab } & \multicolumn{2}{|c|}{$\begin{array}{c}\text { HR From AG Cox } \\
\text { Regression Model } \\
\text { (tocilizumab/etanercept) }^{\mathrm{a}}\end{array}$} \\
\hline & $\begin{array}{l}\text { No. (\%) } \\
\text { With } \\
\text { Event }\end{array}$ & $\begin{array}{l}\text { No. of } \\
\text { Events }\end{array}$ & $\begin{array}{c}\text { Rate per } \\
100 \text { Patient- } \\
\text { Years }\end{array}$ & $\begin{array}{l}\text { No. (\%) } \\
\text { With } \\
\text { Event }\end{array}$ & $\begin{array}{l}\text { No. of } \\
\text { Events }\end{array}$ & $\begin{array}{c}\text { Rate per } \\
100 \text { Patient- } \\
\text { Years }\end{array}$ & Estimate & $95 \% \mathrm{Cl}$ \\
\hline \multicolumn{9}{|l|}{ General AEs } \\
\hline AEs leading to withdrawal of study drug & $105(7)$ & 105 & 2.4 & $120(5)$ & 120 & 2.8 & 1.15 & $0.89-1.49$ \\
\hline $\begin{array}{l}\text { All SAEs } \\
\text { AEs of special interest }\end{array}$ & $356(23)$ & 631 & 14.4 & $421(27)$ & 666 & 15.7 & 1.10 & $0.94-1.28$ \\
\hline Serious infection & $111(7)$ & 139 & 3.2 & $159(10)$ & 190 & 4.5 & 1.39 & $1.08-1.79$ \\
\hline Fatal infection & $8(0.5)$ & 8 & 0.18 & $6(0.4)$ & 6 & 0.14 & 0.76 & $0.26-2.21$ \\
\hline Confirmed gastrointestinal perforation & $1(0.06)$ & 1 & 0.02 & $8(0.5)$ & 8 & 0.19 & 8.43 & $1.06-67.26$ \\
\hline Malignancies (including NMSC) & $38(3)$ & 40 & 0.9 & $38(3)$ & 41 & 1.0 & 1.00 & $0.64-1.58$ \\
\hline Malignancies (excluding NMSC) & $27(2)$ & 28 & 0.6 & $29(2)$ & 30 & 0.7 & 1.09 & $0.64-1.84$ \\
\hline
\end{tabular}

This article is protected by copyright. All rights reserved. 


\begin{tabular}{|c|c|c|c|c|c|c|c|c|}
\hline Significant hypersensitivity ${ }^{b}$ & $17(1)$ & 17 & 0.4 & $10(1)$ & 14 & 0.3 & 0.66 & $0.30-1.46$ \\
\hline Serious hypersensitivity $^{c}$ & $7(0.5)$ & 7 & 0.2 & $4(0.3)$ & 4 & 0.1 & 0.61 & $0.18-2.05$ \\
\hline Serious demyelinating disorders & 0 & 0 & 0 & 0 & 0 & 0 & - & - \\
\hline Serious bleeding events & $13(1)$ & 17 & 0.4 & $19(1)$ & 20 & 0.5 & 1.26 & $0.61-2.62$ \\
\hline Serious hepatic events & $1(0.06)$ & 1 & 0 & $3(0.2)$ & 3 & 0.1 & 2.70 & $0.31-23.43$ \\
\hline Deep venous thrombosis ${ }^{d}$ & $12(0.8)$ & 12 & 0.3 & $9(0.6)$ & 10 & 0.2 & 0.83 & $0.34-2.03$ \\
\hline Pulmonary embolism ${ }^{d}$ & $8(0.5)$ & 8 & 0.2 & $1(0.1)$ & 1 & $(0.06)$ & 0.13 & $0.02-1.04)$ \\
\hline
\end{tabular}

Included are 4373 patient-years in 1542 etanercept-treated patients and 4245 patient-years in 1538 tocilizumab-treated patients.

${ }^{a}$ HRs were calculated using the Andersen-Gill Cox regression model for repeated events, including treatment (tocilizumab/etanercept) as the only covariate and using the robust sandwich-type estimate of variance. Stratification factors are previous exposure to anti-TNF (yes/no) and history of CV events (yes/no). Patients not experiencing an event were censored at last direct contact, capped at the date of treatment discontinuation or the common study end date + 28 days, whichever was earlier.

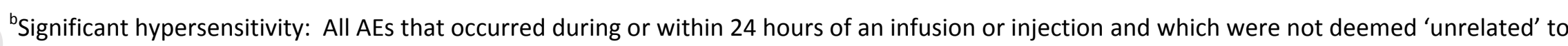
treatment by the investigator, regardless of whether or not they were clinically consistent with hypersensitivity, and led to study treatment discontinuation. 'Serious hypersensitivity: All AEs that occurred during or within 24 hours of an infusion or injection and which were not deemed 'unrelated' to treatment by the investigator, regardless of whether or not they were consistent with hypersensitivity, and were reported as a SAE.

${ }^{\mathrm{d}}$ Deep venous thrombosis and pulmonary embolism were not pre-defined AEs of special interest in the protocol

$\mathrm{AEs}=$ adverse events; $\mathrm{AG}=$ Andersen-Gill; $\mathrm{Cl}=$ confidence interval; $\mathrm{CV}=$ cardiovascular; $\mathrm{HR}=$ hazard ratio; $\mathrm{NMSC}=$ nonmelanomatous skin cancer; $\mathrm{SAEs}=$ serious adverse events; TNF = tumor necrosis factor.

This article is protected by copyright. All rights reserved. 


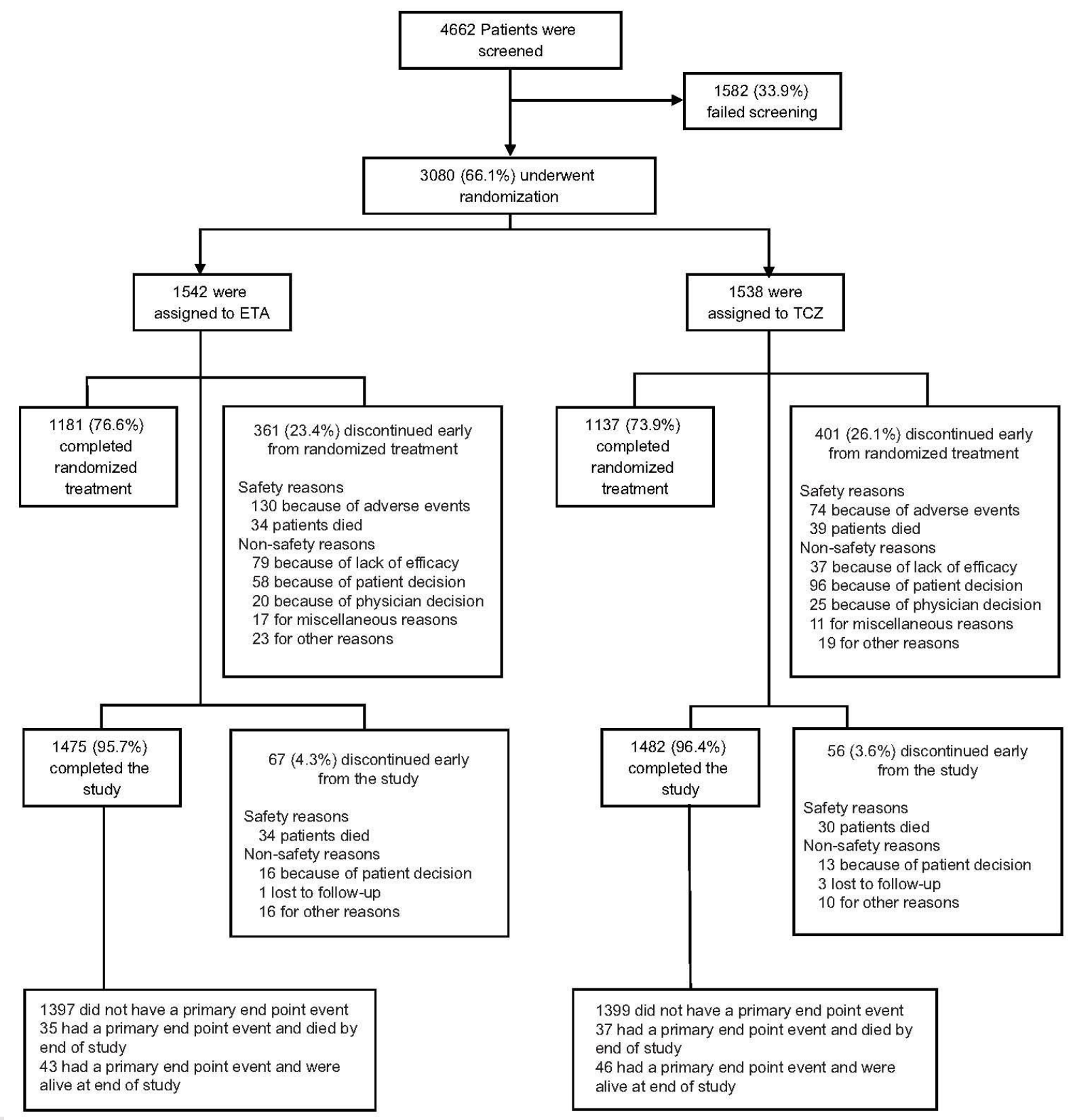

This article is protected by copyright. All rights reserved. 
A. Total Cholesterol

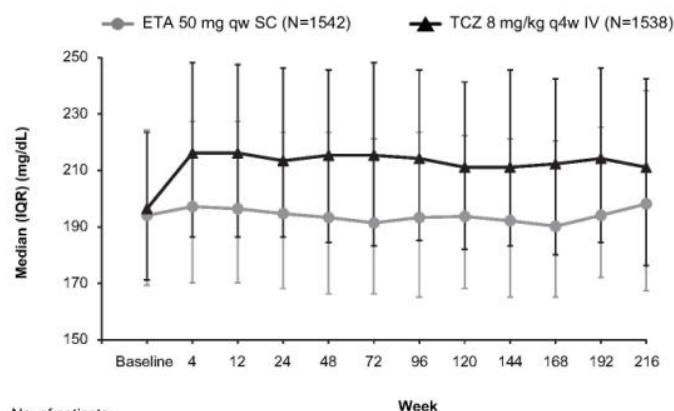
№. of patients

$\begin{array}{lllllllllllll}\text { ETA } & 1541 & 1424 & 1479 & 1458 & 1397 & 1342 & 1311 & 1276 & 1115 & 775 & 399 & 78\end{array}$ $\begin{array}{lllllllllllll}\text { TCZ } & 1538 & 1498 & 1463 & 1419 & 1344 & 1294 & 1254 & 1191 & 1004 & 681 & 315 & 57\end{array}$

\section{B. LDL Cholesterol}

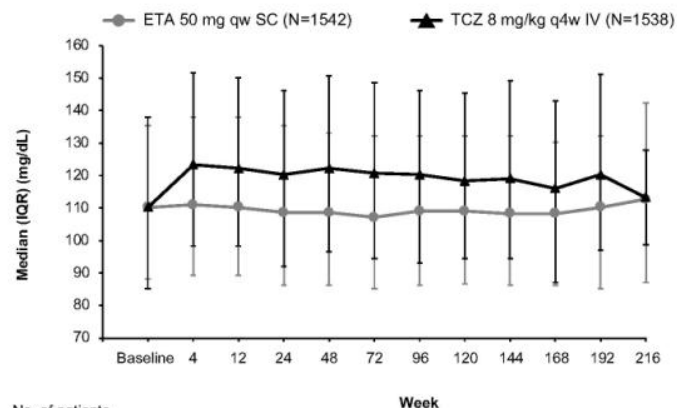

$$
\begin{array}{rllllllllllllll}
\text { No. of patients } & \multicolumn{10}{c}{\text { Week }} \\
\text { ETA } & 1541 & 1423 & 1479 & 1456 & 1396 & 1342 & 1310 & 1276 & 1113 & 775 & 399 & 78 \\
\text { TCZ } & 1537 & 1495 & 1462 & 1419 & 1342 & 1294 & 1253 & 1191 & 1003 & 681 & 315 & 57
\end{array}
$$

\section{HDL Cholesterol}

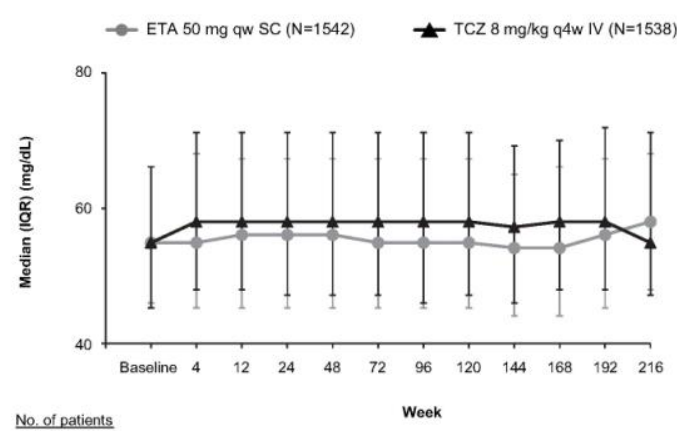

$\begin{array}{lllllllllllll}\text { ETA } & 1540 & 1424 & 1479 & 1458 & 1398 & 1343 & 1311 & 1276 & 1115 & 775 & 399 & 78\end{array}$

$\begin{array}{lllllllllllll}\text { TCZ } & 1537 & 1498 & 1463 & 1419 & 1346 & 1294 & 1254 & 1191 & 1004 & 681 & 315 & 57\end{array}$

\section{Triglycerides}

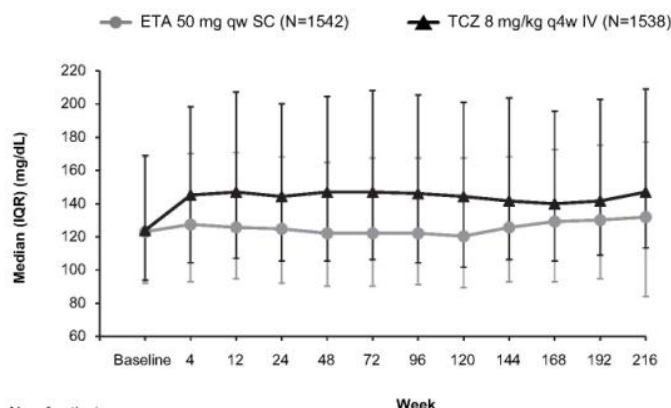
№. of patients

$\begin{array}{lllllllllllll}\text { ETA } & 1541 & 1424 & 1479 & 1458 & 1398 & 1343 & 1311 & 1276 & 1115 & 775 & 399 & 78\end{array}$ $\begin{array}{lllllllllllll}\text { TCZ } & 1538 & 1496 & 1463 & 1419 & 1344 & 1294 & 1253 & 1191 & 1004 & 681 & 315 & 57\end{array}$

This article is protected by copyright. All rights reserved. 